\title{
Novel Approaches to Monitor and Manipulate Single Neurons In Vivo
}

\author{
Michael Brecht, ${ }^{1}$ Michale S. Fee, ${ }^{2}$ Olga Garaschuk, ${ }^{3}$ Fritjof Helmchen, ${ }^{4}$ Troy W. Margrie, ${ }^{6}$ Karel Svoboda,${ }^{7}$ and \\ Pavel Osten ${ }^{5}$ \\ ${ }^{1}$ Department of Neuroscience, Erasmus Medical Center, University Medical Center Rotterdam, 3015 DR Rotterdam, The Netherlands, ${ }^{2}$ Department of Brain \\ and Cognitive Sciences, McGovern Institute, Massachusetts Institute of Technology, Cambridge, Massachusetts 02139, ${ }^{3}$ Institute of Physiology, Ludwig- \\ Maximilians University Munich, 80336 Munich, Germany, Departments of ${ }^{4}$ Cell Physiology and ${ }^{5}$ Molecular Neurobiology, Max Planck Institute for Medical \\ Research, 69120 Heidelberg, Germany, ${ }^{6}$ Department of Physiology, The Wolfson Institute for Biomedical Research, University College London, London \\ WC1E 6BT, United Kingdom, and ${ }^{7}$ Howard Hughes Medical Institute, Cold Spring Harbor Laboratory, Cold Spring Harbor, New York 11743
}

Key words: cortex; patch clamp; in vivo; extracellular recording; two-photon imaging; lentivirus

The complexity of the vertebrate brain poses an enormous challenge to experimental neuroscience. One way of dealing with this complexity has been to investigate different aspects of brain function in widely different preparations, each best suited to address a particular question. Accordingly, cellular questions are typically addressed with intracellular recordings in in vitro preparations such as brain slices or neuronal cultures, whereas network behavior and sensory or motor response properties are analyzed in vivo, often with extracellular recordings. This division of labor has proved to be an experimentally effective strategy. However, although there seems to be no limit to the wealth of data that can be generated in this way, integrating results derived in different preparations comes with its own set of challenges. The enormous difficulties encountered when one attempts to link cellular phenomena such as synaptic plasticity to systems properties such as spatial memory (Martin et al., 2000) have shown us that close collaboration between molecular-cellular and systems neuroscience is required (Tonegawa et al., 2003) and that we need more convergence of experimental techniques to analyze the cellular basis of neural function under more natural conditions. Studying neurons under naturalistic conditions is, however, easier said than done. A return to in vivo preparations will only be successful if we are able to solve the technical problems that led previous researchers to abandon the study of intact brains in the first place. Thus, studying neurons at the cellular level in vertebrate brains is today first and foremost a technological challenge. Here we highlight recent efforts to improve our ability to analyze functions of single neurons in vivo. Given the "mini-review format," we cannot aim for completeness but must focus on the techniques featured in the accompanying mini-symposium without meaning to imply that other novel developments are less significant.

Received Aug. 15, 2004; revised Sept. 1, 2004; accepted Sept. 2, 2004.

Correspondence should be addressed to Michael Brecht, Department of Neuroscience, Erasmus Medical Center, University Medical Center Rotterdam, Dr. Molewaterplein 50, 3015 DR Rotterdam, The Netherlands. E-mail: m.brecht@erasmusmc.nl.

DOI:10.1523/JNEUROSCI.3344-04.2004

Copyright $\odot 2004$ Society for Neuroscience $\quad$ 0270-6474/04/249223-05\$15.00/0

\section{Monitoring neurons with improved cellular resolution}

Structural plasticity and synaptic function

Synapses are the smallest units of organization in neural networks, and they are thought to encode memories. What happens at synapses when we learn? To understand synaptic dynamics in intact animals, it will be necessary to monitor the structure and function of individual synapses in vivo over times of milliseconds to months. This goal has been made attainable by the invention of two-photon laser scanning microscopy (2PLSM) (Denk et al., 1990), which allows imaging in the scattering environment of the intact brain (Denk and Svoboda, 1997). In addition, fluorescent proteins [with their large extinction ratios, quantum efficiencies, and resistance to photobleaching (Tsien, 1998)] are ideal for in vivo imaging and can be genetically targeted to neurons of interest (see below) (Feng et al., 2000). Long-term 2PLSM imaging of green fluorescent protein (GFP)-expressing neurons is used to track structural plasticity in vivo (Grutzendler et al., 2002; Trachtenberg et al., 2002). Whereas dendritic arbors in the adult brain are essentially stable, a fraction of dendritic spines appear and disappear over time periods of days (Trachtenberg et al., 2002). The turnover of dendritic spines is increased after novel sensory experience, suggesting that spine addition and subtraction, together with synapse formation and elimination, is a substrate of experience-dependent plasticity (Trachtenberg et al., 2002) (Fig. 1A). Fluorescent proteins have been engineered to indicate aspects of cellular activity, including $\mathrm{pH}$ changes (Miesenbock et al., 1998), $\mathrm{Ca}^{2+}$ concentration (Miyawaki et al., 1997), membrane potential (Siegel and Isacoff, 1997), and others (Miyawaki, 2003). Thus, aspects of synaptic function such as glutamate release (Oertner et al., 2002) will become accessible in vivo. Two-photon imaging can be easily combined with fluorescence lifetime measurements for quantitative fluorescence resonance energy transfer imaging (So et al., 2000). These techniques may help to decipher the cascades of signal transduction underlying synaptic plasticity in vivo.

\section{Network analysis}

Understanding neuronal computations requires a multilevel approach, and, hence, tools for analysis of network activity need to complement the synaptic and cellular analysis methods. Network 
activity has "microscopic" (individual neurons) and "macroscopic" (neuronal ensembles) aspects, which should preferably be monitored simultaneously. Current techniques [extracellular multielectrode recordings, functional magnetic resonance imaging, imaging of intrinsic optical signals, and voltage-sensitive dye-based imaging (Orbach et al., 1985; Grinvald et al., 1988; Raichle, 1998; Shoham et al., 1999; Nicolelis and Ribeiro, 2002)] are well suited for monitoring activity at the macroscopic level but are restricted in their ability to read out the activity of individual neurons. The divide between the macroscopic and the microscopic brain activity can be bridged by the recently developed multi-cell bolus loading (MCBL) technique, which provides a targeted staining of neuronal populations with calciumsensitive dyes (Stosiek et al., 2003). The cells are stained by a brief bolus injection of a membrane-permeant calcium indicator dye (e.g., Calcium Green-1 AM) into extracellular space and imaged by means of two-photon laser scanning microscopy. This technique allows simultaneous functional analyses of many individual neurons, i.e., a monitoring of brain activity at both the microscopic and macroscopic level (Fig. $1 B$ ). To clarify the identity of the imaged cells, mixtures of a $\mathrm{Ca}^{2+}$-sensitive dye and different cell-specific markers (e.g., an astrocyte marker sulforhodamine 101) (Nimmerjahn et al., 2004) may be used for bolus injections. Identified cells can be further subjected to two-photon targeted patching (TPTP) (Margrie et al., 2003) to measure electrophysiological signals underlying their activity. MCBL is also very useful for monitoring activity in awake, behaving animals (Helmchen et al., 2001; Adelsberger et al., 2004), thus providing a versatile tool for analysis of intact neuronal circuits.

\section{Monitoring neural activity under natural conditions}

Extracellular recordings

Although recent advances in electrode fabrication and microdrive techniques have led to the ability to record from many neurons in freely behaving animals (Bragin et al., 2000; Serruya et al., 2002; Nicolelis et al., 2003), many challenges remain in the effort to develop chronic recording systems with the flexibility and selectivity of acute recording systems. For mechanical stability, implanted multielectrode arrays are usually constructed from electrodes (e.g., microwires) that are optimal for multiunit (multi-cell) rather than single-unit (single-cell) signals (Gray et al., 1995). These signals can be decomposed into putative single-unit signals using spike-sorting techniques, but this method is relatively insensitive to smaller neurons and neurons with low firing rates. To avoid these difficulties, chronic recordings could be made with large numbers of electrodes optimized for single-unit recording that are individually moved in the brain to achieve high-quality single-unit signals on every electrode. We and others have recently made advances toward this goal by developing a motorized microdrive in which electrodes can be individually and remotely manipulated to isolate single neurons (Fee and Leonardo, 2001; Cham et al., 2004).
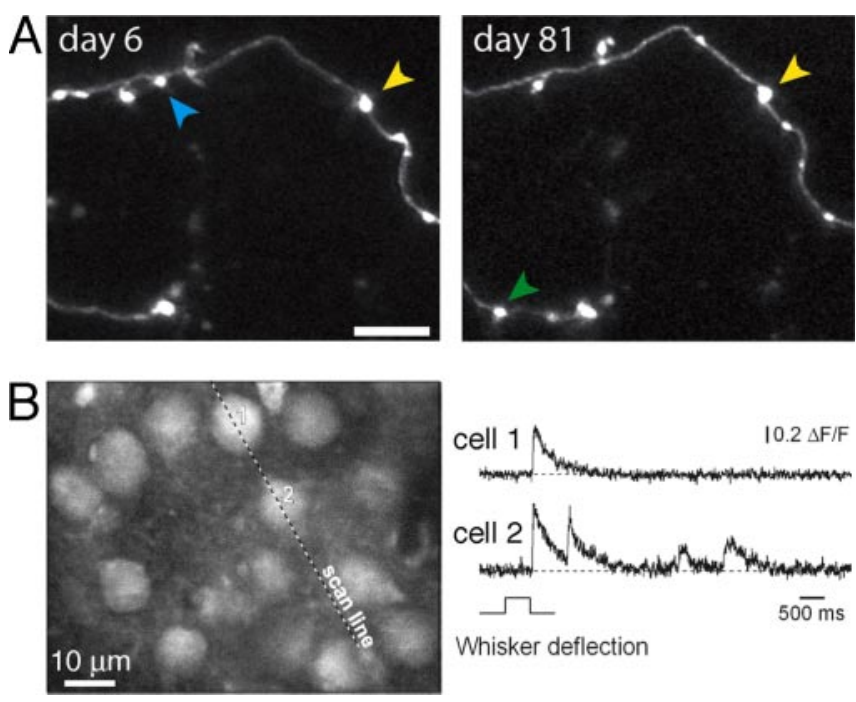

Figure 1. Monitoring neurons with improved cellular resolution. $A$, Long-term in vivo imaging of GFP-expressing axons in the adult mouse barrel cortex. Note gained (e.g., green arrowhead), lost (blue arrowhead), and stable (yellow arrowheads) putative synaptic terminals (A. Holtmaat, personal communication). $B, \mathrm{Ca}^{2+}$ transients evoked by whisker deflection. Left, A highmagnification image of layer 2/3 neurons in vivo (depth, $130 \mu \mathrm{m}$ ) in the barrel cortex of a 13-d-old mouse. Right, Line-scan recordings of $\mathrm{Ca}^{2+}$ transients evoked in two neurons by a deflection of the majority of whiskers on the contralateral side of the mouse's snout. The position of the scanned line and the cells analyzed are indicated (left) (modified from Stosiek et al., 2003).

Another limitation of extracellular recording techniques is that neurons are usually found during recording process by searching for spontaneous spikes as the recording electrode is slowly advanced through the brain. Unfortunately, in some cases,
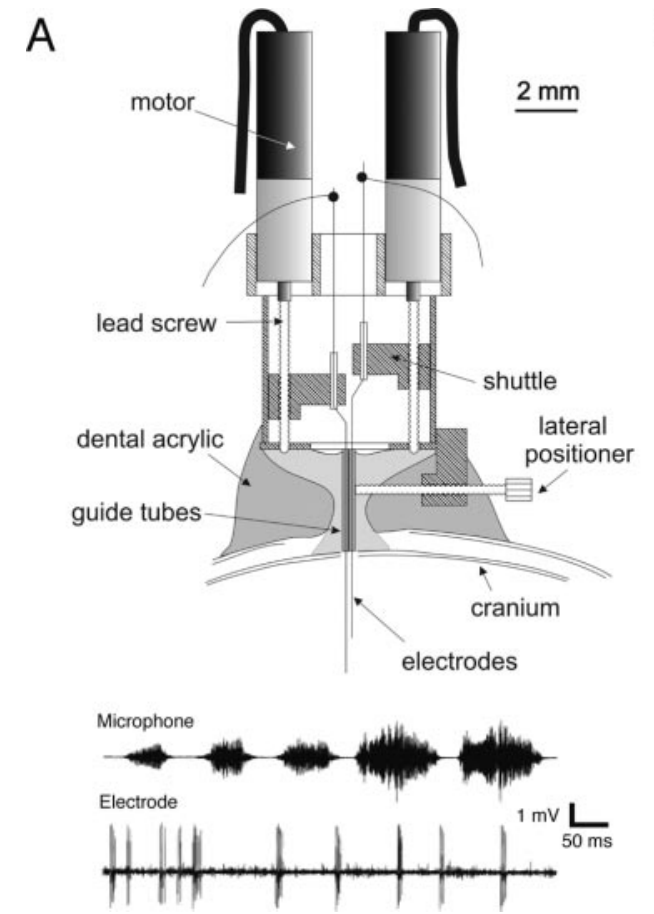
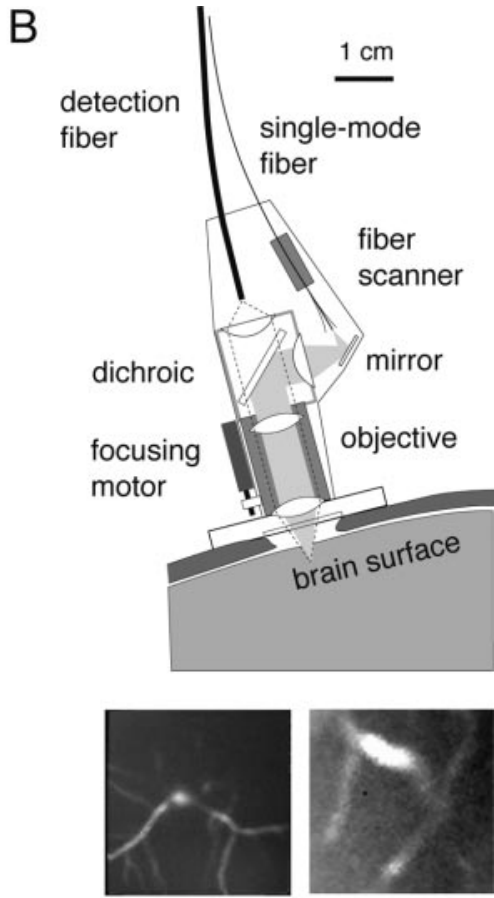

Figure 2. Monitoring neural activity under natural conditions. A, Top, Overview of motorized microdrive. Electrodes are driven independently by small brushless DC motors. Bottom, Sample recording from a single neuron in the nucleus robustus archistriatalis of a zebra finch during singing (adapted from Fee and Leonardo, 2001). B, Top, Principal design of the miniaturized two-photon microscope. Excitation light and fluorescence emission light are guided through optical fibers. The two-photon fiberscope is mounted above a small cranial window and can be carried by freely moving rats. Bottom, Two-photon fiberscope-acquired image of a layer 2 neuron filled with Calcium Green-1 (left) and small blood vessels labeled via tail-vein injection of FITC- dextran (right) (adapted from Helmchen et al., 2001). 

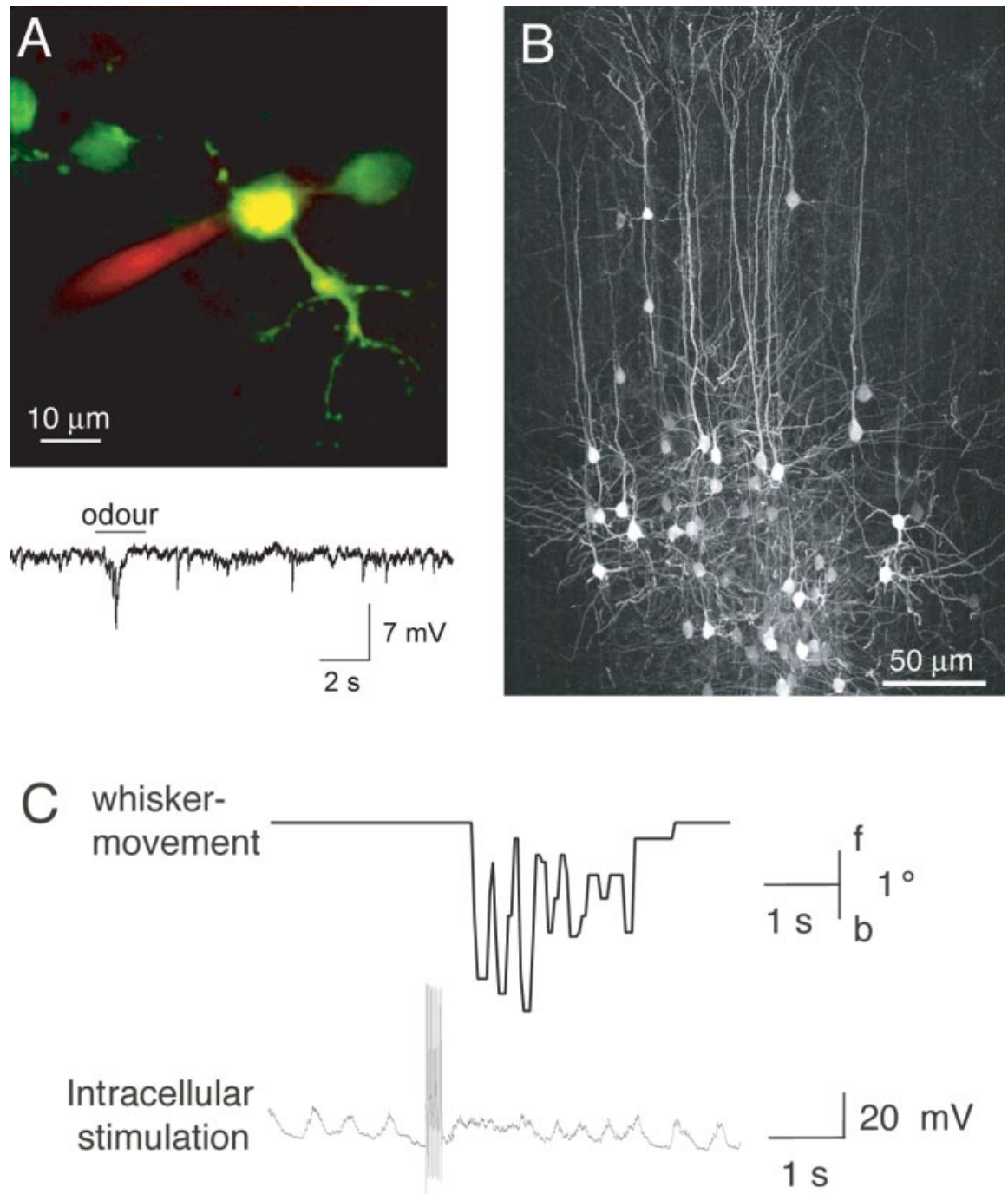

Figure 3. Targeting and manipulating single neurons. A, Top, Targeted whole-cell recording from a calretinin-positive interneuron in the glomerulus layer of the mouse olfactory bulb (postnatal day 21). Image is an overlay of two channels (red and green) showing the illuminated patch pipette filled with Alexa 594 attached to the GFP-labeled interneuron. Bottom, Trace shows membrane potential in response to odor presentation ( $2 \mathrm{sec}$ ). B, Enhanced GFP-expressing lentivirus was injected into the parenchyma of rat layer $2 / 3$ somatosensory cortex (postnatal day 28). After a 2 week expression period, the rat was killed, and enhanced GFP fluorescence was visualized from $100 \mu \mathrm{m}$ paraformaldehyde-fixed brain sections. The image is a maximal projection of 40 confocal sections, separated by $2 \mathrm{~nm}$. C, Intracellular stimulation of the rat primary whisker motor cortex. Top trace, Position of whisker E1 during an intracellular stimulation trial. Bottom trace, Membrane potential recordings during current injection (10 action potentials at $50 \mathrm{~Hz}$ ); the stimulation current is not shown.

neurons exhibit little or no spontaneous activity and cannot be found using this approach. We recently used the classic technique of antidromic activation (Swadlow, 1998) to find premotor neurons in the songbird brain that are completely silent in the awake bird, except when the bird sings. Furthermore, during the song, these neurons generate a single brief burst of spikes (Hahnloser et al., 2002). The discovery of extremely sparse activity in a critical population of premotor neurons in the songbird, as well as other studies (Beloozerova et al., 2003), suggest that a combination of remote manipulation of selective electrodes, and antidromic identification techniques, should be useful to study brain circuitry in freely behaving animals.

\section{Microscope miniaturization}

The development of miniaturized fiber-optic devices for imaging neurons at microscopic resolution in brains of awake, freely moving animals is both an important goal and a major technological challenge. Two types of fiber-optic fluorescence microscopes have been developed. The first type delivers excitation light through a single-core fiber, in which case two-dimensional images are obtained by a scanning mechanism in the microscope headpiece (Delaney and Harris, 1995; Helmchen et al., 2002). Using such a fiberscope, we could resolve neurons and dendrites in cortical layer 2 of anesthetized rats and small blood vessels in freely moving animals (Helmchen et al., 2001) (Fig. $2 B)$. A particular challenge in this case has been the use of two-photon excitation through optical fiber to gain imaging depth (Helmchen et al., 2002). In standard glass fibers, nonlinear effects cause broadening of the ultrafast light pulses and thus reduce two-photon excitation efficiency (Helmchen et al., 2002). Fortunately, radically new types of optical fibers (with hollow air-filled cores) now permit distortion-free propagation of femtosecond pulses (Göbel et al., 2004a). A second type of fiber-optic microscope is based on so-called coherent optical fiber bundles, which contain many thousands of fiber cores and transmit entire images, albeit with low spatial resolution (Hirano et al., 1996; Knittel et al., 2001; Göbel et al., 2004b). Fiber bundles are now available in small diameters and can be attached to small cylindrical lenses suitable for endoscopic imaging. These ultrathin $(<1$ $\mathrm{mm}$ diameter) microscope probes will extend imaging to deeper brain structures (Jung and Schnitzer, 2003; Levene et al., 2004). Microscope miniaturization together with the improvements in methods for fluorescence labeling in vivo (Stosiek et al., 2003) promises to shed light on cellular activity in behaving animals.

\section{Targeting and manipulating single neurons \\ Targeted recordings}

An increasing refinement of recording specificity has been a leading theme in the development of the whole-cell patch-clamp recording technique (Hamill et al., 1981). When combined with differential interference contrast or fluorescence microscopy, the readily visible tip of the patch pipette could be targeted not only to a specific cell genetically manipulated to express a particular fluorophore but also to multiple locations on the same neuron (Stuart et al., 1993). The stability of whole-cell recordings combined with the possibility of rapidly loading fluorescent indicators (Helmchen and Waters, 2002) has therefore made blind whole-cell recordings attractive to in vivo experimentalists (Pei et al., 1991; Jagadeesh et al., 1993). Whole-cell recordings can be obtained from the anesthetized and awake preparation, superficial and deep structures (Margrie et al., 2002), extremely small cells (Chadderton et al., 2004), and even from the dendrites of large cortical neurons (Larkum and Zhu, 2002). One major drawback of blind whole-cell recordings is the random but not necessarily represen- 
tative sampling of neurons, which severely limits the interpretation and the rapidity of data acquisition in neuronally heterogeneous brain regions. Recently, it has become possible to target electrophysiological recordings to genetically labeled neurons in vivo. To this end, two-photon microscopy is used to guide a whole-cell patch pipette filled with a fluorescent dye onto a GFPexpressing neuron in the intact brain (Margrie et al., 2003) (Fig. $3 A$ ). This TPTP technique was initially used to record from the sparsely distributed parvalbumin-positive interneurons in the somatosensory cortex. TPTP experiments on GFP-expressing parvalbumin-positive interneurons provided the first piece of direct evidence in support of a role of electrical coupling in these cells during sensory processing in vivo (Margrie et al., 2003). More recently, TPTP has been used to target cortical cells that have been altered in their intrinsic and synaptic conductances (Komai et al., 2004) by lentivirus-based genetic means (see Single-cell genetics).

\section{Single-cell genetics}

The establishment of novel recording and stimulation techniques makes it highly desirable to develop methods that would combine the optical and electrophysiological single-cell analyses with the genetic means to manipulate individual neurons. Our approach for providing such a methodology, termed "single-cell genetics," is based on stereotactic delivery of lentiviruses (Naldini and Verma, 2000). Lentiviral infection can be titrated to affect small numbers of neurons (on the order of tens to few hundreds) within intact brain networks (Fig. 3B), providing an excellent spatiotemporal control of the onset of the genetic manipulations. Importantly, the lentiviral vector-encompassed genetic information is stably integrated into the host cell genome, allowing for subsequent functional analysis as early as several days and as late as weeks or months after the viral delivery. We optimized the use of the self-inactivating lentiviral vectors (Lois et al., 2002) specifically for combination with cortical physiology, primarily by developing pyramidal neuron-specific expression from synapsin I or $\alpha$-CaMKII $\left(\mathrm{Ca}^{2+} /\right.$ calmodulin-dependent protein kinase II) promoters (Dittgen et al., 2004). Coexpression of GFP and Cre recombinase from these vectors can now be used in mice with floxed genes for creating gene knock-out, whereas coexpression of GFP and double-stranded short-interfering RNAs is an efficient method for gene knock-down in the infected population of pyramidal neurons in the otherwise intact brain (Elbashir et al., 2001; Pfeifer et al., 2001; Licznerski et al., 2003; Qin et al., 2003). The advantages of this approach lie in the ease of its application, as well as in the fact that modification of a small neuronal population avoids lethality of phenotype or activation of compensatory mechanisms that are often associated with standard genetics approaches affecting the whole brain or particular brain regions.

\section{Reverse physiology}

It is unlikely that, if one had access to all the spike trains in all brain cells of an animal of choice, it would be possible to derive mechanistic explanations for the animal's mental events and behavioral performance. Thus, in addition to the correlative analysis of single-neuron activity, we need to study the brain by altering singleneuron activity. On long timescales, such manipulations might be done very elegantly by genetic approaches. On short timescales, microstimulation techniques have been applied (Tehovnik, 1996; Cohen and Newsome, 2004) and have, for example, provided very direct evidence for the involvement of certain sets of cortical neurons in visual and tactile neural representations (Salzman et al., 1990; Romo and Salinas, 2001). The major drawback of microstimulation techniques is their lack of cellular specificity, i.e., the stimulated cel- lular elements are not identified and estimates of the number of stimulated neurons vary by more than an order of magnitude (Tehovnik, 1996). To overcome such limitations, intracellular stimulation has been applied recently in the vibrissa motor cortex (Woody and Black-Cleworth, 1973; Brecht et al., 2004). Quite unexpectedly, we found that single cells could drive noticeable movements. These evoked movements were spatiotemporally complex and varied as function of the lamina of the stimulated neuron (Fig. 3C). We hope to extend this work to a "reverse physiology approach," in which one studies the perceptual and motor correlates of experimentally evoked spikes rather than (as is done conventionally) analyzing the occurrence of spikes as a correlate of external or internal events.

\section{Summary}

As yet, most in vivo records of cellular activity in the vertebrate brain come from unidentified cells, and most manipulations of neural activity affect the whole brain or large populations of neurons in particular brain regions. The combined application of the optical, electrophysiological, and genetic techniques outlined here will allow us to refine our focus to the structure and function of identified neurons in the intact brain. This is the only means by which we can determine with any precision the cellular basis of brain function.

\section{References}

Adelsberger H, Garaschuk OJM, Milos J, Enache A, Konnerth A (2004) In vivo monitoring of calcium waves in behaving mice. FENS Abstr 2:A148.142.

Beloozerova IN, Sirota MG, Swadlow HA (2003) Activity of different classes of neurons of the motor cortex during locomotion. J Neurosci 23:1087-1097.

Bragin A, Hetke J, Wilson CL, Anderson DJ, Engel Jr J, Buzsaki G (2000) Multiple site silicon-based probes for chronic recordings in freely moving rats: implantation, recording and histological verification. J Neurosci Methods 98:77-82.

Brecht M, Schneider M, Sakmann B, Margrie TW (2004) Whisker movements evoked by stimulation of single pyramidal cells in rat motor cortex. Nature 427:704-710.

Chadderton P, Margrie TW, Hausser M (2004) Integration of quanta in cerebellar granule cells during sensory processing. Nature 428:856-860.

Cham JG, Branchaud EA, Nenadic Z, Greger B, Andersen RA, Burdick JW (2004) A semi-chronic motorized microdrive and control algorithm for autonomously isolating and maintaining optimal extracellular action potentials. J Neurophysiol, in press.

Cohen MR, Newsome WT (2004) What electrical microstimulation has revealed about the neural basis of cognition. Curr Opin Neurobiol 14:169-177.

Delaney PM, Harris MR (1995) Fiberoptics in confocal microscopy. In: Handbook of biological confocal microscopy (Pawley JB, ed), pp 515523. New York: Plenum.

Denk W, Svoboda K (1997) Photon upmanship: why multiphoton imaging is more than a gimmick. Neuron 18:351-357.

Denk W, Strickler JH, Webb WW (1990) Two-photon laser scanning fluorescence microscopy. Science 248:73-76.

Dittgen T, Licznerski P, Grinevich V, Cetin A, Laudenklos S, Seeburg PH, Osten P (2004) Single cell genetics: a novel approach to study neuronal functions in vivo. FENS Abstr 2:A190.196.

Elbashir SM, Harborth J, Lendeckel W, Yalcin A, Weber K, Tuschl T (2001) Duplexes of 21-nucleotide RNAs mediate RNA interference in cultured mammalian cells. Nature 411:494-498.

Fee MS, Leonardo A (2001) Miniature motorized microdrive and commutator system for chronic neural recording in small animals. J Neurosci Methods 112:83-94.

Feng G, Mellor RH, Bernstein M, Keller-Peck C, Nguyen QT, Wallace M, Nerbonne JM, Lichtman JW, Sanes JR (2000) Imaging neuronal subsets in transgenic mice expressing multiple spectral variants of GFP. Neuron 28:41-51.

Göbel W, Nimmerjahn A, Helmchen F (2004a) Distortion-free delivery of 
femtosecond pulses from a Ti:sapphire laser through a hollow core photonic crystal fiber. Opt Lett 29:1285-1287.

Göbel W, Kerr JND, Nimmerjahn A, Helmchen F (2004b) A miniaturized two-photon microscope using a flexible coherent fiber bundle and a gradient-index lens objective. Opt Lett, in press.

Gray CM, Maldonado PE, Wilson M, McNaughton B (1995) Tetrodes markedly improve the reliability and yield of multiple single-unit isolation from multi-unit recordings in cat striate cortex. J Neurosci Methods 63:43-54.

Grinvald A, Frostig RD, Lieke E, Hildesheim R (1988) Optical imaging of neuronal activity. Physiol Rev 68:1285-1366.

Grutzendler J, Kasthuri N, Gan WB (2002) Long-term dendritic spine stability in the adult cortex. Nature 420:812-816.

Hahnloser RH, Kozhevnikov AA, Fee MS (2002) An ultra-sparse code underlies the generation of neural sequences in a songbird. Nature 419:65-70.

Hamill OP, Marty A, Neher E, Sakmann B, Sigworth FJ (1981) Improved patch-clamp techniques for high-resolution current recording from cells and cell-free membrane patches. Pflügers Arch 391:85-100.

Helmchen F, Waters J (2002) $\mathrm{Ca}^{2+}$ imaging in the mammalian brain in vivo. Eur J Pharmacol 447:119-129.

Helmchen F, Fee MS, Tank DW, Denk W (2001) A miniature headmounted two-photon microscope. High-resolution brain imaging in freely moving animals. Neuron 31:903-912.

Helmchen F, Tank DW, Denk W (2002) Enhanced two-photon excitation through optical fiber by single-mode propagation in a large core. Appl Opt 41:2930-2934.

Hirano M, Yamashita Y, Miyakawa A (1996) In vivo visualization of hippocampal cells and dynamics of $\mathrm{Ca}^{2+}$ concentration during anoxia: feasibility of a fiber-optic plate microscope system for in vivo experiments. Brain Res 732:61-68.

Jagadeesh B, Wheat HS, Ferster D (1993) Linearity of summation of synaptic potentials underlying direction selectivity in simple cells of the cat visual cortex. Science 262:1901-1904.

Jung JC, Schnitzer MJ (2003) Multiphoton endoscopy. Opt Lett 28:902-904.

Knittel J, Schnieder L, Buess G, Messerschmidt B, Possner T (2001) Endoscope-compatible confocal microscope using a gradient index-lens system. Opt Commun 188:267-273.

Komai S, Waters J, Licznerski P, Denk W, Brecht M, Osten P (2004) The role of active conductances in the development of Receptive Field properties in the rat barrel cortex studied with single-cell genetics and TPTP. Soc Neurosci Abstr 30:58.8.

Larkum ME, Zhu JJ (2002) Signaling of layer 1 and whisker-evoked $\mathrm{Ca}^{2+}$ and $\mathrm{Na}^{+}$action potentials in distal and terminal dendrites of rat neocortical pyramidal neurons in vitro and in vivo. J Neurosci 22:6991-7005.

Levene MJ, Dombeck DA, Kasischke KA, Molloy RP, Webb WW (2004) In vivo multiphoton microscopy of deep brain tissue. J Neurophysiol 91:1908-1912.

Licznerski P, Dittgen T, Seeburg PH, Osten P (2003) Lentivirus-based vectors for gene silencing in neurons. Soc Neurosci Abstr 29:325.7.

Lois C, Hong EJ, Pease S, Brown EJ, Baltimore D (2002) Germline transmission and tissue-specific expression of transgenes delivered by lentiviral vectors. Science 295:868-872.

Margrie TW, Brecht M, Sakmann B (2002) In vivo, low-resistance, wholecell recordings from neurons in the anaesthetized and awake mammalian brain. Pflügers Arch 444:491-498.

Margrie TW, Meyer AH, Caputi A, Monyer H, Hasan MT, Schaefer AT, Denk W, Brecht M (2003) Targeted whole-cell recordings in the mammalian brain in vivo. Neuron 39:911-918.

Martin SJ, Grimwood PD, Morris RG (2000) Synaptic plasticity and memory: an evaluation of the hypothesis. Annu Rev Neurosci 23:649-711.

Miesenbock G, De Angelis DA, Rothman JE (1998) Visualizing secretion and synaptic transmission with $\mathrm{pH}$-sensitive green fluorescent proteins. Nature 394:192-195.

Miyawaki A (2003) Visualization of the spatial and temporal dynamics of intracellular signaling. Dev Cell 4:295-305.
Miyawaki A, Llopis J, Heim R, McCaffery JM, Adams JA, Ikura M, Tsien RY (1997) Fluorescent indicators for $\mathrm{Ca}^{2+}$ based on green fluorescent proteins and calmodulin. Nature 388:882-887.

Naldini L, Verma IM (2000) Lentiviral vectors. Adv Virus Res 55:599-609.

Nicolelis MA, Ribeiro S (2002) Multielectrode recordings: the next steps. Curr Opin Neurobiol 12:602-606.

Nicolelis MA, Dimitrov D, Carmena JM, Crist R, Lehew G, Kralik JD, Wise SP (2003) Chronic, multisite, multielectrode recordings in macaque monkeys. Proc Natl Acad Sci USA 100:11041-11046.

Nimmerjahn A, Kirchhoff F, Kerr JND, Helmchen F (2004) Sulforhodamine 101 as a specific marker of astroglia in the neocortex in vivo. Nature Methods, in press.

Oertner TG, Sabatini BL, Nimchinsky EA, Svoboda K (2002) Facilitation at single synapses probed with optical quantal analysis. Nat Neurosci 5:657-664.

Orbach HS, Cohen LB, Grinvald A (1985) Optical mapping of electrical activity in rat somatosensory and visual cortex. J Neurosci 5:1886-1895.

Pei X, Volgushev M, Vidyasagar TR, Creutzfeldt OD (1991) Whole cell recording and conductance measurements in cat visual cortex in-vivo. NeuroReport 2:485-488.

Pfeifer A, Brandon EP, Kootstra N, Gage FH, Verma IM (2001) Delivery of the Cre recombinase by a self-deleting lentiviral vector: efficient gene targeting in vivo. Proc Natl Acad Sci USA 98:11450-11455.

Qin XF, An DS, Chen IS, Baltimore D (2003) Inhibiting HIV-1 infection in human $\mathrm{T}$ cells by lentiviral-mediated delivery of small interfering RNA against CCR5. Proc Natl Acad Sci USA 100:183-188.

Raichle ME (1998) Behind the scenes of functional brain imaging: a historical and physiological perspective. Proc Natl Acad Sci USA 95:765-772.

Romo R, Salinas E (2001) Touch and go: decision-making mechanisms in somatosensation. Annu Rev Neurosci 24:107-137.

Salzman CD, Britten KH, Newsome WT (1990) Cortical microstimulation influences perceptual judgements of motion direction. Nature 346:174-177.

Serruya MD, Hatsopoulos NG, Paninski L, Fellows MR, Donoghue JP (2002) Instant neural control of a movement signal. Nature 416:141-142.

Shoham D, Glaser DE, Arieli A, Kenet T, Wijnbergen C, Toledo Y, Hildesheim R, Grinvald A (1999) Imaging cortical dynamics at high spatial and temporal resolution with novel blue voltage-sensitive dyes. Neuron 24:791-802.

Siegel MS, Isacoff EY (1997) A genetically encoded optical probe of membrane voltage. Neuron 19:735-741.

So PT, Dong CY, Masters BR, Berland KM (2000) Two-photon excitation fluorescence microscopy. Annu Rev Biomed Eng 2:399-429.

Stosiek C, Garaschuk O, Holthoff K, Konnerth A (2003) In vivo two-photon calcium imaging of neuronal networks. Proc Natl Acad Sci USA 100:7319-7324.

Stuart GJ, Dodt HU, Sakmann B (1993) Patch-clamp recordings from the soma and dendrites of neurons in brain slices using infrared video microscopy. Pflügers Arch 423:511-518.

Swadlow HA (1998) Neocortical efferent neurons with very slowly conducting axons: strategies for reliable antidromic identification. J Neurosci Methods 79:131-141.

Tehovnik EJ (1996) Electrical stimulation of neural tissue to evoke behavioral responses. J Neurosci Methods 65:1-17.

Tonegawa S, Nakazawa K, Wilson MA (2003) Genetic neuroscience of mammalian learning and memory. Philos Trans R Soc Lond B Biol Sci 358:787-795.

Trachtenberg JT, Chen BE, Knott GW, Feng G, Sanes JR, Welker E, Svoboda $\mathrm{K}$ (2002) Long-term in vivo imaging of experience-dependent synaptic plasticity in adult cortex. Nature 420:788-794.

Tsien RY (1998) The green fluorescent protein. Annu Rev Biochem 67:509-544.

Woody CD, Black-Cleworth P (1973) Differences in excitability of cortical neurons as a function of motor projection in conditioned cats. J Neurophysiol 36:1104-1116. 